\title{
Shock Waves in Capillary Collapse of Colloids: A Model System for Two-Dimensional Screened Newtonian Gravity
}

\author{
J. Bleibel, ${ }^{1,4}$ S. Dietrich,,${ }^{1,2}$ A. Domínguez, ${ }^{3}$ and M. Oettel ${ }^{4}$ \\ ${ }^{1}$ Max-Planck-Institut für Intelligente Systeme, Heisenbergstr. 3, 70569 Stuttgart, Germany \\ ${ }^{2}$ Institut für Theoretische und Angewandte Physik, Universität Stuttgart, Pfaffenwaldring 57, 70569 Stuttgart, Germany \\ ${ }^{3}$ Física Teórica, Universidad de Sevilla, Apdo. 1065, 41080 Sevilla, Spain \\ ${ }^{4}$ Institut für Physik, WA 331, Johannes Gutenberg Universität Mainz, 55099 Mainz, Germany
}

(Received 6 May 2011; published 16 September 2011)

\begin{abstract}
Using Brownian dynamics simulations, density functional theory, and analytical perturbation theory we study the collapse of a patch of interfacially trapped, micrometer-sized colloidal particles, driven by longranged capillary attraction. This attraction is formally analogous to two-dimensional (2D) screened Newtonian gravity with the capillary length $\hat{\lambda}$ as the screening length. Whereas the limit $\hat{\lambda} \rightarrow \infty$ corresponds to the global collapse of a self-gravitating fluid, for finite $\hat{\lambda}$ we predict theoretically and observe in simulations a ringlike density peak at the outer rim of a disclike patch, moving as an inbound shock wave. Possible experimental realizations are discussed.
\end{abstract}

DOI: 10.1103/PhysRevLett.107.128302

PACS numbers: 82.70.Dd, 47.11.Mn, 47.40. $-\mathrm{x}$

The dynamics of matter under the influence of longranged attractions is studied intensively in several branches of physics [1], in particular, with respect to inherent instabilities. Most prominently, the structure formation in the universe is understood as the consequence of an instability in self-gravitating matter, and cosmological theories in conjunction with numerical simulations have been successfully applied to explain the dynamical formation of clusters, galaxies, or dark matter halos on large scales [2]. More recently, other systems with gravitational-like attractions have been investigated, including seemingly unrelated phenomena like bacterial chemotaxis $[3,4]$ or capillary-driven clustering in colloids trapped at fluid interfaces $[5,6]$. In these systems the interaction is effectively cut off beyond a finite range, albeit much larger than the mean interparticle separation.

In a self-gravitating fluid any homogeneous mass distribution is unstable with respect to small fluctuations on sufficiently large scales [7] (Jeans's instability). In systems with a cutoff gravitational-like attraction, this instability only occurs below a critical temperature $[4,5]$. As the range of the interaction is scaled [6] from infinity down to a microscopic length like the size of the particles, the dynamical evolution of the instability crosses over from gravitational collapse to spinodal decomposition. A standard theoretical approach to the gravitational collapse in cosmology is the so-called cold collapse approximation (see, e.g., Ref. [8]), within which any force other than gravity (in particular the thermal pressure of the fluid) is neglected altogether. The view on applications to other physical situations raises the natural question of how the phenomenology of this scenario is affected by a nonvanishing thermal pressure and a large but finite range of attractions and specifically how the crossover to the spinodal decomposition scenario occurs.
Colloidal particles with radii in the micrometer range, which are trapped at a fluid interface, lend themselves to study this issue. Their weight results in a force $f$ on each particle perpendicular to the interface which deforms it and gives rise to long-ranged capillary interactions between the particles. For large colloid center-to-center distances $d$, the leading interaction term (dominating the collective collapse dynamics) is a pair interaction with the potential [9] $V(d)=\left[f^{2} /(2 \pi \gamma)\right] K_{0}(d / \hat{\lambda})$ with the modified Bessel function $K_{0}$, the capillary length $\hat{\lambda}(\sim O(\mathrm{~mm}))$, and the interfacial tension $\gamma$. For $d<\hat{\lambda}$ this reduces to 2D Newtonian gravity, $V_{0}(d) \sim \ln d$, whereas for $d>\hat{\lambda}$ it decreases exponentially. This soft matter system is of particular interest because the range $\hat{\lambda}=\sqrt{\gamma /\left(g \Delta \rho_{m}\right)}$ is tunable via the dependence of $\gamma$ on temperature $\hat{T}$ and the concentration of surfactants or through the mass density difference $\Delta \rho_{m}$ between the two fluid phases forming the interface; $g$ is Earth's gravitational acceleration.

Main results.-We study the time evolution of the 2D particle number density $\hat{\varrho}(\hat{\mathbf{r}}=\{x, y\}, \hat{t})$ of an initially circular patch of radius $L$ with particles of radius $R$ on the flat interface with a homogenous density $\hat{\varrho}_{0}$. The range is measured by the dimensionless parameter $\lambda=\hat{\lambda} / L$ and we introduce the effective, dimensionless temperature

$$
T=\frac{\gamma k_{\mathrm{B}} \hat{T}}{f^{2} \hat{\varrho}_{0} L^{2}} .
$$

For $\hat{\lambda} \rightarrow \infty, T$ is the ratio of the thermal to the (mean-field) attractive inner energy of the patch because each particle interacts with $N \approx \hat{\varrho}_{0} L^{2}$ other particles. For $\hat{\lambda}<L$, the number reduces to $N \approx \hat{\varrho}_{0} \hat{\lambda}^{2}$ and this ratio approximately equals $\left(1+\lambda^{-2}\right) T$. Figure 1 summarizes qualitatively the dynamic phases we have found. If $T$ is large enough (above the black line), the capillary attraction cannot confine the 


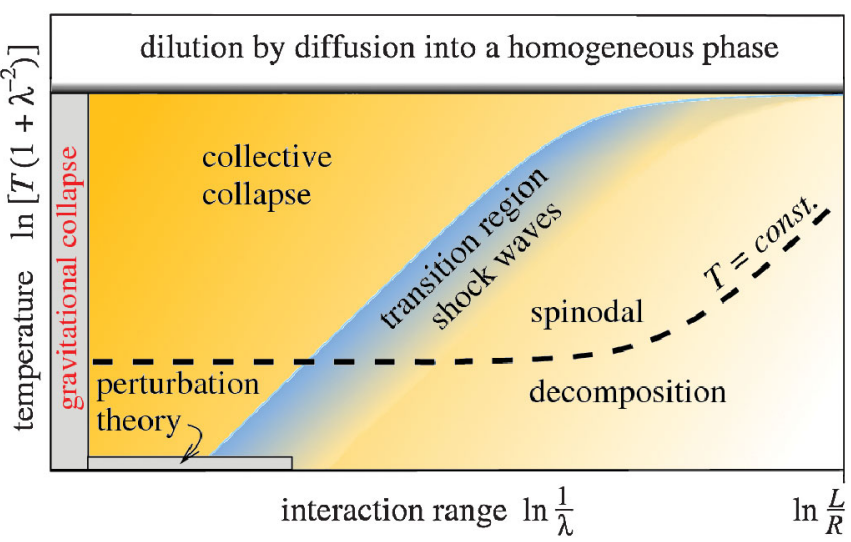

FIG. 1 (color). Proposed sketch of dynamical regimes for a circular patch of radius $L$ with particles of radius $R$ as a function of the range $\lambda=\hat{\lambda} / L$ of the interaction and a rescaled effective temperature $T\left(1+\lambda^{-2}\right)$ [see Eq. (1) and below]. The rescaling factor for $T$ leads to a horizontal border separating the collapse and dilution regimes. Neglecting a possible temperature dependence of $\hat{\lambda}$, isotherms are parallel to the dashed black line. The transition region is bounded approximately by the line for which linear stability theory for an infinite homogeneous distribution $[5,6]$ predicts that the fastest growing density mode has a wave number $2 \pi / L$.

particles to the circular patch, so that it becomes more and more diluted as time progresses. If $T$ is small (dashed line) the patch collapses, but how this proceeds depends on $\lambda$. In the limit $\lambda \rightarrow \infty$ (gravitational collapse), the fastest growing modes span the patch and the evolution is dominated by the collapse of the structure as a whole. In the opposite limit $\lambda \rightarrow R$ (spinodal decomposition), the fastest growing modes have a characteristic length scale well below $L$ and the evolution is dominated by the coarsening of these domains. We have explored the transition region between these limits using perturbation theory, Brownian dynamics (BD) simulations, and dynamic density functional theory (DDFT). Accordingly, the most prominent feature of this transition is a density peak forming at the outer rim of the

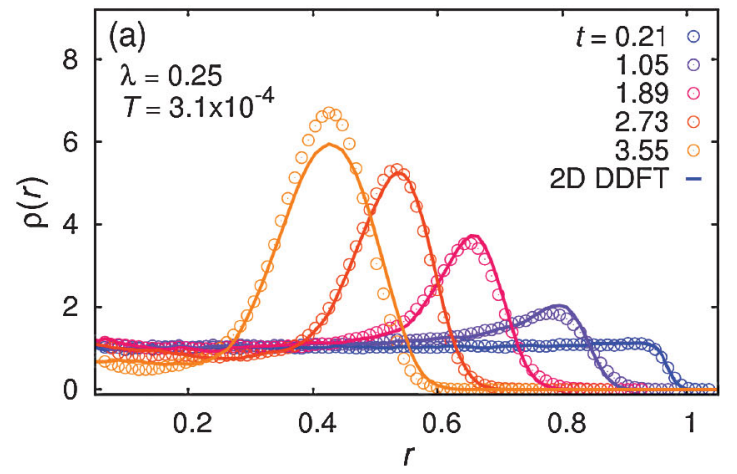

collapsing circular patch which exhibits properties of a shock wave. The latter is defined by the crossing of the characteristic curves of a differential equation. For Eq. (2) below, in the presence of radial symmetry, these characteristics are the trajectories of rings of particles. Rings with initially larger radii travel faster than those with smaller radii and, upon crossing, form a density singularity as $T \rightarrow 0$ [Fig. 2(a)]. This feature becomes more pronounced with $\lambda$ becoming smaller, because the time for building up the shock wave diminishes relative to the collapse time of the whole patch (Fig. 3). On the other hand, for smaller $\lambda$ additional small clusters form in the interior of the patch and the shock wave amplitude is reduced [Fig. 2(b)].

Theory.-We consider a 2D fluid within a mean-field approximation appropriate for the long-ranged, capillary interactions and take into account nonzero temperature and short-ranged interactions through a pressure equation of state $\hat{p}(\hat{\varrho}, \hat{T})$ [5], containing the effects of short-ranged interactions such as hard or soft cores and subleading terms in the capillary forces. The particle dynamics is assumed to be in the overdamped regime with $\Gamma$ as the associated interfacial mobility of the particles and hydrodynamic interactions are neglected. The relevant time scale of collapse is Jeans's time $\mathcal{T}=\gamma /\left(\Gamma f^{2} \hat{\varrho}_{0}\right)$. We note that the time it takes a particle to diffuse a distance $L$ by Brownian motion alone is $\sim L^{2} /\left(\Gamma k_{\mathrm{B}} \hat{T}\right)=\mathcal{T} / T$. Dimensionless variables are introduced as $\varrho=\hat{\varrho} / \hat{\varrho}_{0}, p=\hat{p} /\left(k_{\mathrm{B}} \hat{T} \hat{\varrho}_{0}\right)$, $\mathbf{r}=\hat{\mathbf{r}} / L$, and $t=\hat{t} / \mathcal{T}$ together with Eq. (1). Mass conservation reads [5]

$$
\frac{\partial \varrho}{\partial t}=-\nabla \cdot(\varrho \nabla U[\varrho]-T \nabla p)
$$

with the dimensionless potential of capillary interaction $U[\varrho(\mathbf{r})]=[1 /(2 \pi)] \int d \mathbf{r}^{\prime} \varrho\left(\mathbf{r}^{\prime}\right) K_{0}\left(\left|\mathbf{r}-\mathbf{r}^{\prime}\right| / \lambda\right)$. For idealized point particles the pressure is $p_{\text {id }}=\varrho$; we have also considered a fluid of hard discs of radius $R$, described by [10] $p_{\text {hd }}(\varrho)=\varrho\left(\varrho_{c}+\varrho\right) /\left(\varrho_{c}-\varrho\right)$, where $\varrho_{c}=\left(2 \sqrt{3} \hat{\varrho}_{0} R^{2}\right)^{-1}$ is the dimensionless density of close

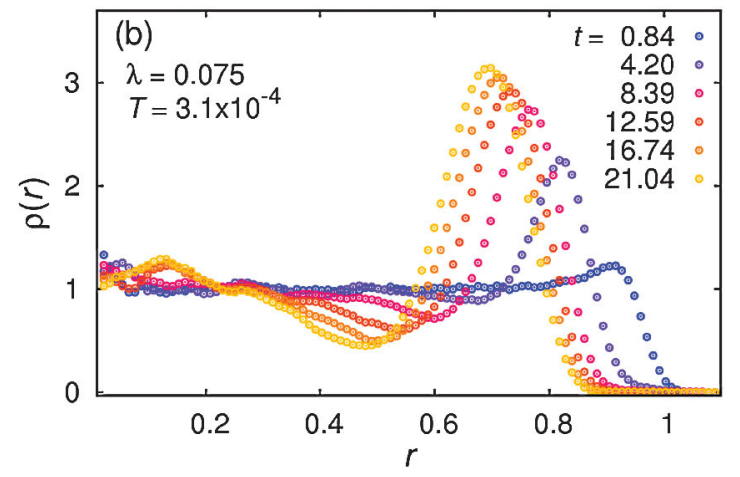

FIG. 2 (color). Evolution of the radial density profile for $T=3.1 \times 10^{-4}$ (for further parameters, see the main text). Panel (a): $\lambda=0.25$, comparison between 2D DDFT (colored lines), and BD (symbols). Panel (b): $\lambda=0.075$, only BD (DDFT results omitted for clarity). Unlike as in (a), small transient peaks distinct from the one at the outer rim are observed due to clustering near the center of the collapsing disc. 

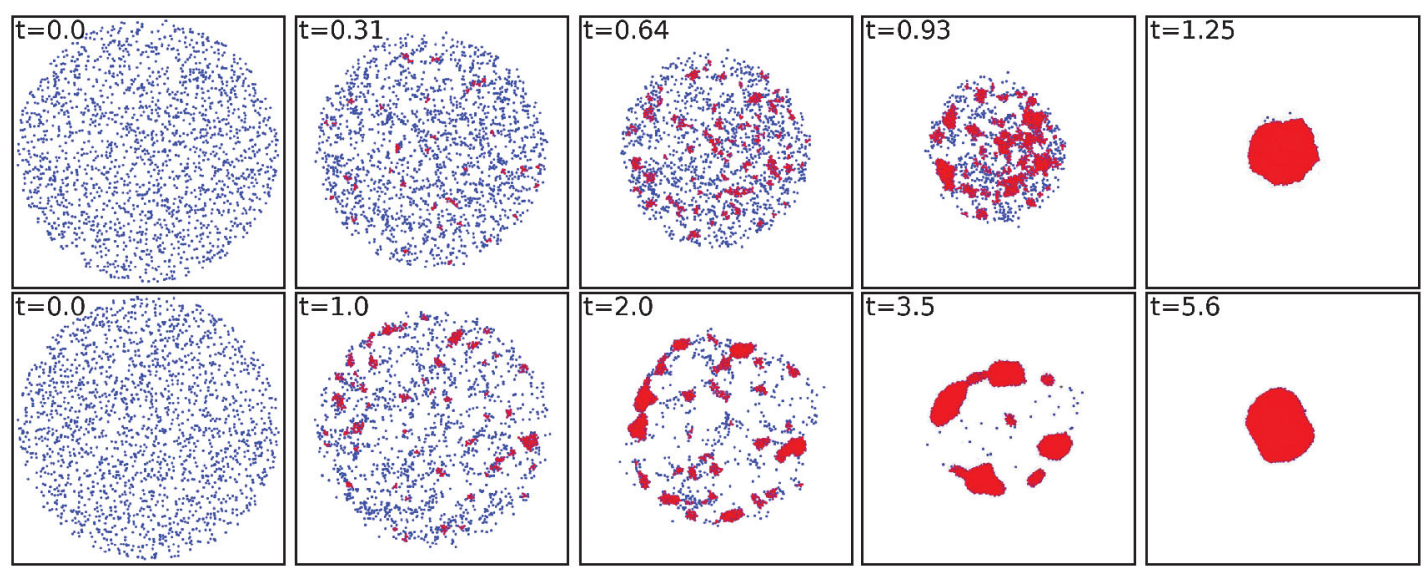

FIG. 3 (color). Snapshots from BD simulations for temperature $T=3.1 \times 10^{-4}$ and $\lambda=1.5$ (upper row) or $\lambda=0.25$ (lower row). Particle distributions in each column have the same radial extent. Clusters (i.e., particles with at least 3 neighbors within a distance of $3.25 R$ ) are depicted in red. For $\lambda=1.5$ the global collapse appears to be faster than the formation of individual smaller clusters. For $\lambda=0.25$, small clusters predominantly form at the outer rim and collectively move towards the center.

packing. Equation (2) can be viewed as a simple DDFT for this system [5] which, given the long range of the attractions, is expected to hold at least for scales much larger than $R$ and to describe correctly the collective aspects of the dynamics. Deviations are likely to occur for smaller scales and are mainly attributable to the local density approximation, i.e., the crude form of the term $\propto \nabla p$ for the short-ranged interactions. Here, improvement could be achieved using more sophisticated expressions from DFT.

The cold collapse (CC) scenario [formally $T=0$ in Eq. (2)] is best studied using Lagrangian coordinates: the characteristic curves of Eq. (2) are the radial trajectories of infinitesimally thin rings of particles, assuming that the initial radial symmetry is preserved by the mean-field evolution. If the initial radius is $r_{0}$, the trajectory is described by a time dependent mapping $r=r_{\mathrm{La}}\left(r_{0}, t\right)$. The Jacobian of this mapping provides the density field in Lagrangian coordinates:

$$
\frac{1}{\varrho_{\mathrm{La}}\left(r_{0}, t\right)}=\frac{r_{\mathrm{La}}}{r_{0}} \frac{\partial r_{\mathrm{La}}}{\partial r_{0}}, \quad\left(r_{0} \leq 1\right)
$$

The mapping is well defined and invertible as long as two rings do not cross. If two rings cross, the Jacobian vanishes and the density field exhibits a singularity. For $\lambda \rightarrow \infty$, the CC approximation yields [5,11] $r_{\mathrm{La}}=r_{0} \sqrt{1-t}(t \leq 1)$ and $\varrho_{\mathrm{La}}\left(r_{0}, t\right)=1 /(1-t)$. Thus, the initial homogeneity inside the patch is preserved and a singularity arises at $t=1$ (i.e., at Jeans's time), when all the rings reach the center simultaneously. For finite $\lambda$ we have applied perturbation theory in terms of $1 / \lambda$ leading to [6]

$$
\begin{aligned}
\left(\frac{r_{\mathrm{La}}}{r_{0}}\right)^{2}= & 1-t+\frac{2-4 \gamma_{\text {Euler }}-r_{0}^{2}-\ln \left(4 \lambda^{2}\right)}{(4 \lambda)^{2}} t(2-t) \\
& -\frac{2 \ln \left(4 \lambda^{2}\right)}{(4 \lambda)^{2}}(1-t)^{2} \ln |1-t|,
\end{aligned}
$$

$$
\frac{1}{\varrho_{\mathrm{La}}\left(r_{0}, t\right)}=\left(\frac{r_{L a}}{r_{0}}\right)^{2}-t(2-t)\left(\frac{r_{0}}{4 \lambda}\right)^{2}
$$

This result predicts the formation of an overdensity for the outermost ring $\left(r_{0}=1\right)$ which becomes singular $\left(1 / \varrho_{\mathrm{La}}=0\right)$ at a time $t_{s} \approx 1+\ln (1.44 \lambda) /(2 \lambda)^{2}$ when it reaches a radius $r_{s}=r_{\mathrm{La}}\left(1, t_{s}\right) \approx 1 /(4 \lambda)$. (The singularity is a consequence of the CC approximation; it is actually regularized into a density peak moving like a shock wave by the term $\propto \nabla p$.) Thus, with decreasing $\lambda$ the collapse singularity occurs later and at larger radii. Intuitively, for decreasing $\lambda$ the range of the interaction is confined to smaller regions around any point of the disc. In addition the outer rim of the disc experiences no balancing pull from the outside, so that there the first overdensity builds up, which then attracts more and more particles.

Numerical methods and discussion.-In order to test this theoretical analysis we have performed simulations and solved Eq. (2) numerically. Simulation parameters were chosen to reflect the conditions in an actual experimental realization [5] (patch size $L=1.83 \mathrm{~mm}$, particle radius $R=10 \mu \mathrm{m}$, capillary potential depth $f^{2} /(2 \pi \gamma)=$ $0.89 k_{\mathrm{B}} \hat{T}$, particle mobility $\Gamma=3 \pi \eta_{\text {water }} R$ with $\eta_{\text {water }}$ being the water viscosity at room temperature, and the particle hard core realized by the repulsive part of the Lennard-Jones potential [12]). The simulations were carried out with $N=1804$ particles using BD [6,13] and the radial density profile was obtained through angular and ensemble (120 runs) averages. Numerical solutions were obtained either through a numerical integration of Eq. (2) with enforced radial symmetry or through a particle-based (Lagrangian) integration scheme of the full 2D equation (labeled as 2D DDFT) $[6,14]$. In this latter case, the density field is probed by a discrete number of (fictitious) particles which follow the characteristic curves of Eq. (2) and the 
density profile is obtained like the profiles from BD simulations.

We find that the value $\lambda=1.5$ (i.e., $\hat{\lambda} \approx 2.7 \mathrm{~mm}$, which is the capillary length of the air-water interface at ambient conditions) is at the limit of applicability of the perturbation theory: the DDFT solutions for $T=0$ confirm the occurrence of the singularity at the predicted values $r_{s} \approx 0.16, t_{s} \approx 1.1$ and that the formation of the singularity indeed slows down and occurs at larger values of $r_{s}$ with decreasing $\lambda\left(r_{s} \approx 0.69, t_{s} \approx 1.7\right.$ for $\left.\lambda=0.25\right)$. The DDFT solutions at nonzero $T=O\left(10^{-3}-10^{-5}\right)$ show that, as for $T=0$, an overdensity peak forms at the rim while traveling inwards with increasing amplitude, but the development into a singularity is inhibited.

The results from the BD simulations confirm this and provide further details about the dynamical evolution, see Figs. 2 and 3. For $\lambda=0.25$ at $T=3.1 \times 10^{-4}$, in contrast to the collapse at larger $\lambda$, a ring-shaped densified zone forms which is composed of a number of smaller clusters. The 2D DDFT solutions agree well with the BD simulation data for all times. This indicates that the features we are analyzing are not affected by the details of the microscopic correlations, the effect of which can be taken into account through the macroscopic pressure term. Upon decreasing $\lambda$ further, the formation of even more individual clusters inside the disc is observed, as a prelude to the spinodal decomposition scenario. For the particular value $\lambda=0.075$ the averaged radial density profile still exhibits the collective behavior of the shock wave at the outer rim albeit with a smaller amplitude. Additionally, transient peak structures for smaller radii also become visible and are eventually absorbed by the shock wave traveling inwards.

The phenomenology of local clustering due to small initial fluctuations is also visible in the azimuthal direction, both in the BD simulations and in the 2D DDFT solution. In both cases the initial conditions break the radial symmetry, which is expected to be recovered by an ensemble average.

Concerning a possible experimental realization, a suitable value of $T$ can be arranged using colloids with $R \sim 10 \mu \mathrm{m}$ [5]. Observation of the ring-shaped density buildup and the ensuing shock wave requires $\lambda / L \leqq 0.25$, corresponding to $L \gtrsim 10 \mathrm{~mm}$ for an air-water interface. A likely relevant issue for experiments is the role of hydrodynamic interactions. We have carried out simulations incorporating these on the Rotne-Prager level (as formulated in Ref. [15]) for the specific case of particles with a contact angle close to zero (i.e., just touching the interface). Our results indicate that the qualitative features of the evolution discussed here are not affected and the collapse is simply accelerated. This is in line with the experimentally observed enhanced colloid self-diffusion due to hydrodynamic interactions in such a system [16].
Summary and conclusions. - We have studied the collapse of a homogeneous, circular patch of colloidal particles trapped at a fluid interface by means of analytical perturbation theory, Brownian dynamics simulations, and dynamical density functional-like theories. The capillary attraction is formally analogous to two-dimensional gravity with a tunable cutoff length $\lambda$. We find that a finite value of $\lambda$ strongly influences the collapse features. While for $\lambda \rightarrow \infty$ the evolution is dominated by the global collapse of the patch, a large but finite $\lambda$ induces the formation of a ringlike overdensity which quickly becomes singular in the limit of a vanishing pressure force (i.e., zero temperature or cold collapse). A nonvanishing pressure regularizes the singularity into a collapsing shock wave. System parameters can be chosen such that these spatiotemporal structures can be realized in experiments with micrometer-sized colloids. Furthermore, this system appears to be ideally suited to investigate the transition from Jeans's gravitational instability $(\lambda \rightarrow \infty)$ to a spinodal instability $(\lambda \sim$ colloid radius).

Financial support by the DFG through SFB-TR6 "Colloids in External Fields" (Project N01), by DAADPPP and by the Spanish Government through Grants No. FIS2008-01339 (partially financed by FEDER funds) and No. AIB2010DE-00263 is acknowledged.

[1] A. Campa, T. Dauxois, and S. Ruffo, Phys. Rep. 480, 57 (2009).

[2] V. Springel et al. Nature (London) 435, 629 (2005).

[3] E. F. Keller and L. A. Segel, J. Theor. Biol. 26, 399 (1970).

[4] P. H. Chavanis and L. Delfini, Phys. Rev. E 81, 051103 (2010).

[5] A. Domínguez, M. Oettel, and S. Dietrich, Phys. Rev. E 82, 011402 (2010).

[6] J. Bleibel, A. Domínguez, M. Oettel, and S. Dietrich (unpublished).

[7] J. H. Jeans, Phil. Trans. R. Soc. A 199, 1 (1902).

[8] V. Sahni and P. Coles, Phys. Rep. 262, 1 (1995).

[9] M. Oettel and S. Dietrich, Langmuir 24, 1425 (2008).

[10] E. L. Grossman, T. Zhou, and E. Ben-Naim, Phys. Rev. E 55, 4200 (1997).

[11] P. H. Chavanis and C. Sire, Phys. Rev. E 83, 031131 (2011).

[12] J. D. Weeks, D. Chandler, and H. C. Andersen, J. Chem. Phys. 54, 5237 (1971).

[13] M. P. Allen and D. J. Tildesley, Computer Simulation of Liquids (Clarendon, New York, 1989).

[14] N. Y. Gnedin and L. Hui, Mon. Not. R. Astron. Soc. 296, 44 (1998).

[15] B. Cichocki, M. L. Ekiel-Jezewska, G. Nägele, and E. Wajnryb, Europhys. Lett. 67, 383 (2004).

[16] K. Zahn, J. M. Mendez-Alcaraz, and G. Maret, Phys. Rev. Lett. 79, 175 (1997). 